\title{
Rovalpituzumab Tesirine
}

\author{
National Cancer Institute
}

\section{Source}

National Cancer Institute. Rovalpituzumab Tesirine. NCI Thesaurus. Code C107678.

An antibody-drug conjug ate (ADC) containing a humanized IgG1 monoclonal antibody (MAb) directed against the delta-like protein 3 (DLL3), conjug ated to the cytotoxic pyrrolobenzodiazepine (PBD) dimer D6.5 (SC-DR002) via a maleimide-containing linker with an eight-carbon polyethylene glycol spacer and a cathepsin B-cleavable valinealanine dipeptide, with potential antineoplastic activity. The MAb moiety of rovalpituzumab tesirine selectively binds to DLL3 on tumor cell surfaces. Upon internalization of the ADC, the dipeptide linker is cleaved and D6.5 is released. Then the imine groups of the PBD moiety bind to the N2 positions of guanines on opposite strands of DNA. This induces DNA strand breaks, inhibits DNA replication, leads to G2/M cell cycle arrest, induces cell death, and inhibits the proliferation of DLL3-overexpressing tumor cells. DLL3, a membrane protein that binds to Notch receptors and regulates Notch-mediated signaling and gene transcription, is overexpressed by certain cancers but is rarely expressed by normal, healthy cells. 